Pacific Journal of Mathematics

CONDUCTOR, PROJECTIVITY AND INJECTIVITY 


\title{
CONDUCTOR, PROJECTIVITY AND INJECTIVITY
}

\section{WOLMER V. VASCONCELOS}

\begin{abstract}
Here we discuss the role of the conductor of a ring extension vis-a-vis the descent of projectivity and injectivity. Regarding the former, the first result says that an injective homomorphism of commutative rings descends projectivity if it does so modulo the conductor. The cheap version-that with noetherian hypotheses-of the descent of projectivity by a finite homomorphism due to Gruson then follows easily. A carbon copy-with the natural modification-of the descent of injectivity is also proved.
\end{abstract}

The statement of the results follows very closely the lines of similar work of Ferrand ([2]) on flat modules rather than those of the remarkable [3].

1. Conductor and projectivity. Throughout rings will be commutative with identity element. The price to lift the restriction of commutativity would be to load the exposition with expressions like "two-sided ideal", "bi-module", etc., without any real gain, in view of the fact that for the applications the commutativity is critical.

The main result of this section is, almost word for word, the projective analogue of [2]. Rather than using the results already obtained there, at no cost, we will provide complete proofs based on simple calculations.

THEOREM 1.1. Let $h: A \rightarrow B$ be an injective homomorphism of rings, $I$ an ideal of $A$ and $E$ an $A$-module. Then $E$ is $A$-projective if and only if $B \otimes_{A} E$ is B-projective and $E / I E$ is $A / I$-projective in the following cases:

(i) I is also a B-ideal.

(ii) $I$ is nilpotent.

Proof. (i) (1) $\operatorname{Tor}_{1}^{A}(A / I, E)=0$ :

We must show that the natural map $I \otimes_{A} E \rightarrow E$ is injective. This follows from the commutative diagram

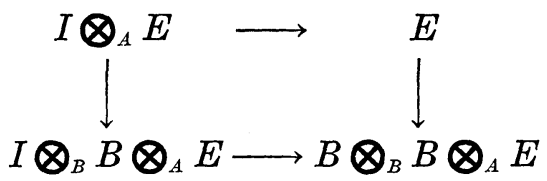

where the vertical map on the left is the natural identification while 
the lower horizontal map is injective by the $B$-flatness of $B \otimes_{A} E$.

(2) $\operatorname{Tor}_{1}^{A}(B, E)=0$ :

Let

$$
0 \longrightarrow G \stackrel{j}{\longrightarrow} F \longrightarrow E \longrightarrow 0
$$

be exact with $F A$-free. By tensoring it with $B$ we get (from now on unadorned tensor products are taken over $A$ )

$\left({ }^{*}\right) \quad 0 \longrightarrow \operatorname{Tor}_{1}^{A}(B, E) \longrightarrow B \otimes G \stackrel{1 \otimes^{j}}{\longrightarrow} B \otimes F \longrightarrow B \otimes E \longrightarrow 0$.

As $B \otimes E$ is $B$-projective, this sequence splits piecemeal; by tensoring it with $B / I$ over $B$, we get the exact sequence

$$
\begin{aligned}
0 \longrightarrow B / I \otimes_{B} \operatorname{Tor}_{1}^{A}(B, E) \longrightarrow B / I \otimes_{B} B \otimes G \\
\longrightarrow B / I \otimes_{B} B \otimes F \longrightarrow B / I \bigotimes_{B} B \otimes E \longrightarrow 0
\end{aligned}
$$

which can also be written

$$
\begin{aligned}
0 \longrightarrow \operatorname{Tor}_{1}^{A}(B, E) / I . \operatorname{Tor}_{1}^{A}(B, E) \longrightarrow B / I \otimes G / I G \\
\longrightarrow B / I \otimes F / I F \longrightarrow B / I \otimes E / I E \longrightarrow 0 .
\end{aligned}
$$

By (1) it follows then that $\operatorname{Tor}_{1}^{A}(B, E)=I$. $\operatorname{Tor}_{1}^{A}(B, E)$. Let now $x \in$ $\operatorname{Tor}_{1}^{A}(B, E)$ : we can write $x=\sum a_{i} \cdot x_{i}$ with $a_{i} \in I, x_{i} \in \operatorname{Tor}_{1}^{A}(B, E) \subset$ $B \otimes G$. It follows thus that $x$ lies in the image of $G$ in $B \otimes G$. But in the diagram

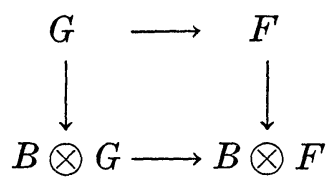

the right vertical map is injective as $h: A \rightarrow B$ is injective and $F$ is $A$-free. We then have $x=0$.

(3) $\left(^{*}\right)$ splits:

Let $\phi$ be a splitting for $\left(^{* *}\right)$, i.e., $\phi(1 \otimes j)=1$. On the other hand, let $\psi$ be a splitting of

$$
0 \longrightarrow G / I G \stackrel{j^{\prime}}{\longrightarrow} F / I F \longrightarrow E / I E \longrightarrow 0 \text {. }
$$

The projectivity of $F$ yields then a map $\theta: F \rightarrow G$ such that $\theta j=1+g$, with $g: G \rightarrow I G$.

From the product

$$
(1-(1 \otimes \theta)(1 \otimes j))(\phi(1 \otimes j)-1)=0
$$

one gets 


$$
((1 \otimes g) \phi+(1 \otimes \theta))(1 \otimes j)=1 .
$$

If we restrict the map $(1 \otimes g) \phi+(1 \otimes \theta)$ to $F$, we get $(1 \otimes g) \phi+\theta) j=1$ where $(1 \otimes g) \phi+\theta$ is actually a map from $F$ into $G$. This completes the proof of (i).

(ii) Say $I^{n}=(0)$ and let $I_{i}=\left(I^{i} \cdot B\right) \cap A$. Then $I_{i}^{2} \subset I_{i+1}$ and $I_{n}=$ (0). By passing to $A / I_{n-1}\left(\hookrightarrow B / I_{n-1}\right.$. $\left.B\right)$ we reduce the question, by induction, to the case $I^{2}=(0)$.

(1) $\operatorname{Tor}_{1}^{A}(A / I, E)=0$ :

This can be read off the diagram

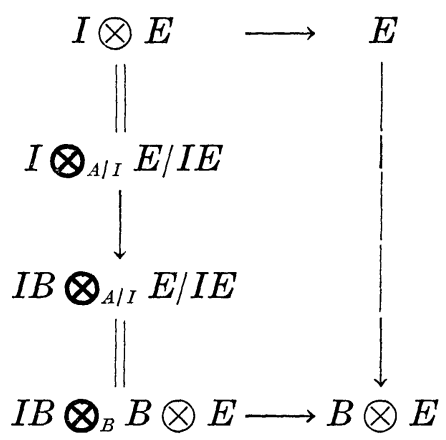

where the left vertical map is injective by the $A / I$-flatness of $E / I E$ while tho lower horizontal map is injective by the $B$-flatness of $B \otimes E$.

(2) $\left({ }^{*}\right)$ splits:

Tensor $\left(^{*}\right)$ with $A / I$ and get $\phi: F \rightarrow G$ such that $\phi j=1+g, g: G \rightarrow$ IG. As $g^{2}=0,(1-g) \phi$ provides the desired splitting map.

REMARKs. (a) In (ii) above it is enough that $I$ be $T$-nilpotent, for it follows from [3, p. 60] that $E$ is flat and the argument in (ii) yields a splitting. (b) If $A$ is artinian, with $I$ the radical of $A$, one has that any injective homomorphism descends projectivity. (c) With $k$ a field and $A=k\left[x_{i}^{2}, x_{i}^{3}, i=1,2, \cdots\right], B=k\left[x_{i}{ }^{\prime} s\right]$ the conductor $I$ of $B$ in $A$ is such that $A / I=k$. Thus the inclusion $A \rightarrow B$ descends projectivity.

CoRollary 1.2. Let $A, B$ be commutative rings under the conditions of (i) above, and let $E$ be an A-module. If $B \otimes E$ (and resp. $E / I E)$ is finitely generated over $B$ (resp.over $A / I$ ), then $E$ is finitely generated over $A$. (Descent of finiteness)

Proof. Pick $n$ large enough such that there is a sequence

$$
A^{n} \longrightarrow E \longrightarrow C \longrightarrow 0
$$

such that $B \otimes C=0$ and $C / I C=0$. Then $C$ is $A$-projective of trace 
ideal, say, J. However the trace of $B \otimes C$ is then $J B$. Since $h: A \rightarrow$ $B$ is injective, this implies $C=0$.

THEOREM 1.3. Let $A$ be a noetherian ring, $h: A \rightarrow B$ an injective homomorphism of rings and $B$ finitely generated as $A$-module. Let $E$ be an $A$-module; if $B \otimes E$ is B:projective then $E$ is A-projective.

Proof. It follows the path of [2]. Let $I$ be a largest ideal of $A$ such that $E / I E$ is not $A / I$-projective. If $I \neq \sqrt{I}=J, E / J E$ is $A / J$-projective and $B / J B \otimes E$ is also $B / J B$-projective $(A / J \subset B / J B)$ and by Theorem 1.1 we get a contradiction. Thus we may assume that $A$ is reduced and such that $E / L E$ is $A / L$-projective for each ideal $L \neq(0)$. We have the canonical inclusion $A \rightarrow A^{\prime}=\Pi\left(A / P_{i}\right), P_{i}$ running through the minimal primes. Let $B^{\prime}=\Pi\left(B / P_{i} B\right)$; then $A^{\prime} \otimes E$ is $A^{\prime}$-projective. However the conductor of $A^{\prime} / A$ is $\neq(0)$ and thus by Theorem $1.1 E$ is $A$-projective.

Assume then $A$ to be a domain. We may take $B=A[y]$ and also a domain. Let $a y^{n}+\cdots+b=0$ be a least degree equation satisfied by $y$. Then with $z=a y A \rightarrow B_{0}=A[z] \rightarrow A[y], B_{0}$ is a free extension of $A$ and hence it is enough to show that $B_{0} \otimes E$ is $B_{0}$-projective. From the hypothesis on $A$ it follows that for every ideal $M \neq(0)$ of $B_{0}, M \cap A \neq(0)$ and $B_{0} / M B_{0} \otimes E$ is $B_{0} / M B_{0}$-projective. Also, since the conductor of $B / B_{0}$ is nonzero, a final application of Theorem 1.1 yields the desired conclusion.

2. Conductor and injectivity. Now we discuss the injective analogue of Theorem 1.1. The existence of injective envelopes is quite essential for the proof.

TheOREM 2.1. Let $h: A \rightarrow B$ be an injective homomorphism of rings, $I$ an ideal of $A$ and $E$ an $A$-module. Then $E$ is A-injective if and only if $\operatorname{Hom}_{A}(B, E)$ is $B$-injective and $\operatorname{Hom}_{A}(A / I, E)$ is $A / I$ injective in the following cases:

(i) $I$ is also a B-ideal.

(ii) $I$ is nilpotent.

Proof. (i) (1) $\operatorname{Ext}_{A}^{1}(A / I, E)=0$ :

Let

$(* * *)$

$$
0 \longrightarrow \stackrel{j}{\longrightarrow} F \stackrel{\pi}{\longrightarrow} C \longrightarrow 0
$$

be exact with $F$ an injective envelope of $E$. It yields

$$
\begin{aligned}
0 \longrightarrow \operatorname{Hom}(A / I, E) \longrightarrow \operatorname{Hom}(A / I, F) \longrightarrow \operatorname{Hom}(A / I, C) & \longrightarrow \operatorname{Ext}^{1}(A / I, E) \longrightarrow 0
\end{aligned}
$$


(where the lower $A$ 's are dropped without risk of confusion). But $\operatorname{Hom}(A / I, F)={ }_{I} F=\{x \in F, I x=0\}$ is the injective envelope of ${ }_{I} E$ as an $A / I$-module and thus ${ }_{I} C=\operatorname{Ext}^{1}(A / I, E)$. We also have the diagram

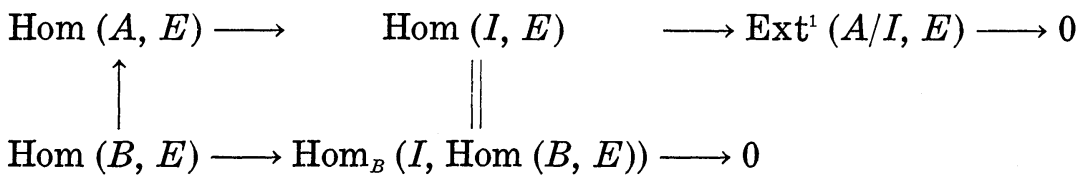

with the lower sequence exact by the $B$-injectivity of $\operatorname{Hom}(B, E)$. It thus follows that ${ }_{I} C=\operatorname{Ext}^{1}(A / I, E)=0$.

(2) $\operatorname{Ext}^{1}(B, E)=0$ :

The sequence

$$
\begin{aligned}
0 \longrightarrow \operatorname{Hom}(B, E) \longrightarrow \operatorname{Hom}(B, F) \longrightarrow \operatorname{Hom}(B, C) \\
\longrightarrow \operatorname{Ext}^{1}(B, E) \longrightarrow 0
\end{aligned}
$$

splits piecemeal as $B$-modules. Applying $\operatorname{Hom}_{B}(B / I,-)$ to it we get

$$
\begin{aligned}
0 \longrightarrow \operatorname{Hom}(B / I, E) \longrightarrow \operatorname{Hom}(B / I, F) \longrightarrow \operatorname{Hom}(B / I, C) \\
\longrightarrow \operatorname{Hom}_{B}\left(B / I, \operatorname{Ext}^{1}(B, E)\right) \longrightarrow 0 \text {. }
\end{aligned}
$$

Since ${ }_{I} C=0,{ }_{I} \operatorname{Ext}^{1}(B, E)=0$ also. Let $\alpha \in \operatorname{Hom}(B, C)$; say $\alpha(1)=c$. Pick $b \in F$ with $\pi(b)=c$ and choose $\beta: A \rightarrow F$ such that $\beta(1)=b$. Let now $\phi: B \rightarrow F$ extend $\beta$. We claim that $\pi \phi=\alpha$. For $a \in I, x \in F$, $a(\pi \phi-\alpha)(x)=\pi \phi(a x)-\alpha(a x)=0$. Thus $I$. image $(\pi \phi-\alpha)=0$ and $\pi \phi=\alpha$ as ${ }_{i} C=0$.

(3) $(* * *)$ splits:

Consider the exact sequences

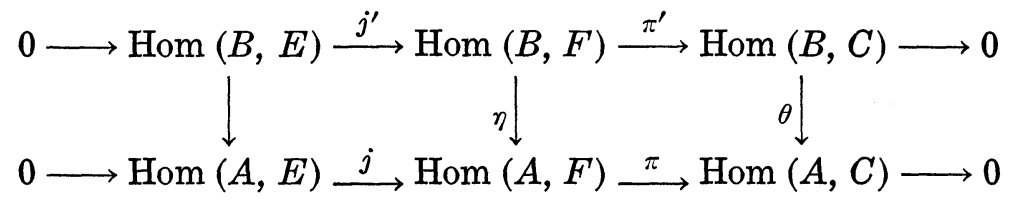

where $\eta$ and $\theta$ are onto by the injectivity of $F$. Let $\phi$ be a splitting for the upper sequence; let $c \in C$ and $\alpha \in \operatorname{Hom}(B, C)$ be such that $\theta(\alpha)=C$ that is, $\alpha$ is such that $\alpha(1)=c$. Define $\psi(c) \in \operatorname{Hom}(A, F)$ to be $\eta(\phi(\alpha))$. We claim that $\psi$ is well defined and provides a splitting for the lower sequence. Assume first that $\alpha(1)=0$ : then $I . \alpha(B)=0$ and thus $\alpha=0$, and $\psi$ is well defined. Next, we have $\pi(\phi(c))=$ $\pi(\eta(\phi(\alpha)))=\theta\left(\pi^{\prime}(\phi(\alpha))\right)=c$.

(ii) Reduce first to the case $I^{2}=(0)$. It is enough now to show that $\operatorname{Ext}^{1}(A / I, E)=0$ for ${ }_{I} E$ is essential in ${ }_{I} F$ and thus ${ }_{I} C=0$ implies $C=0$. Just read this off the commutative diagram 


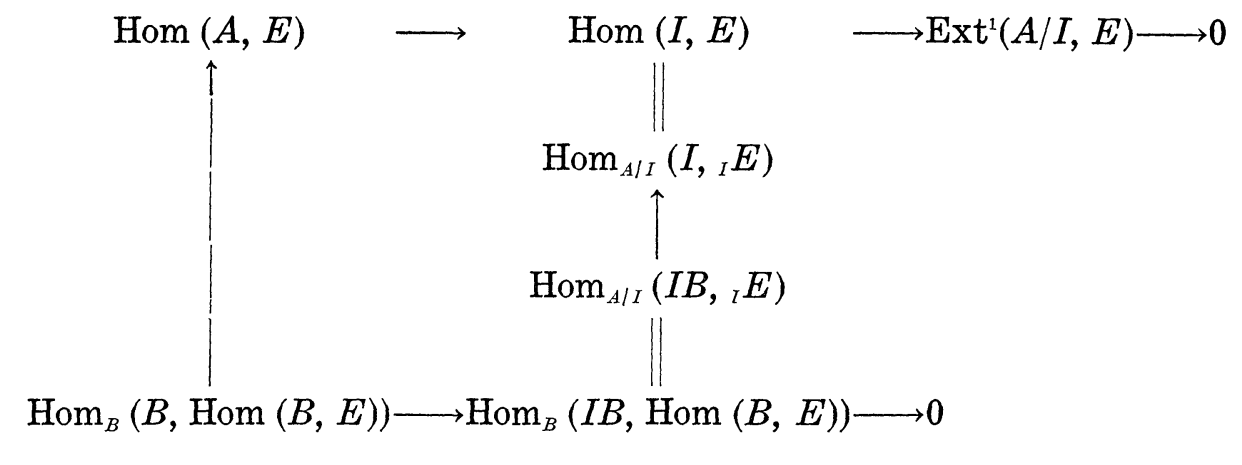

where the right vertical map is surjective by the $A / I$-injectivity of ${ }_{I} E$ and the lower horizontal map is exact by the $B$-injectivity of $\operatorname{Hom}(B, E)$.

CoRollary 2.2. Let $h: A \rightarrow B$ be an injective homomorphism of rings, $I$ the conductor of $B / A$. Then if $A / I$ and $B$ are both noetherian rings and $B / I$ is finitely generated as an $A$-module, $A$ is noetherian (and hence $B$ is finitely generated as $A$-module).

Proof. According to [1, p. 60] it is enough to show that a direct $\operatorname{sum} E=\bigoplus E_{i}$ of injective $A$-modules is injective. Clearly $\operatorname{Hom}\left(A / I, \oplus E_{i}\right)$ is $A / I$-injective. If $f \in \operatorname{Hom}\left(B, \oplus E_{i}\right), f(I) \subset f(A)$ and the finite generation of $B / I$ implies that $f(B)_{i}$ (=projection of $f(B)$ in $\left.E_{i}\right)$ is trivial for almost all $i$ 's. Thus $\operatorname{Hom}\left(B, \oplus E_{i}\right)=\oplus \operatorname{Hom}\left(B, E_{i}\right)$ and Theorem 2.1 takes over.

\section{REFERENCES}

1. H. Bass, Injective dimension in noetherian rings, Trans. Amer. Math. Soc., 102 (1962), 18-29.

2. D. Ferrand, Descent de la platitude par un homomorphisme fini., C. R. Acad. Sc. Paris, 269 (1969), 946-949.

3. L. Gruson and M. Raynaud, Critères de platitude et de projectivité, Inventines Math., 13, (1971), 1-89.

Received May 25, 1972. This research was partially supported by NSF Grant GP-19995. 


\section{PACIFIC JOURNAL OF MATHEMATICS}

\section{EDITORS}

D. Gilbarg and J. Milgram

Stanford University

Stanford, California 94305

\author{
R. A. Beaumont \\ University of Washington \\ Seattle, Washington 98105
}

J. DuGUNDJI

Department of Mathematics

University of Southern California

Los Angeles, California 90007

Richard ARENS

University of California

Los Angeles, California 90024

\section{ASSOCIATE EDITORS}
E. F. BECKENBACH
B. H. NeumanN
F. WOLF
K. YosHIDA

\section{SUPPORTING INSTITUTIONS}

UNIVERSITY OF BRITISH COLUMBIA

CALIFORNIA INSTITUTE OF TECHNOLOGY

UNIVERSITY OF CALIFORNIA

MONTANA STATE UNIVERSITY

UNIVERSITY OF NEVADA

NEW MEXICO STATE UNIVERSITY

OREGON STATE UNIVERSITY

UNIVERSITY OF OREGON

OSAKA UNIVERSITY

\author{
UNIVERSITY OF SOUTHERN CALIFORNIA \\ STANFORD UNIVERSITY \\ UNIVERSITY OF TOKYO \\ UNIVERSITY OF UTAH \\ WASHINGTON STATE UNIVERSITY \\ UNIVERSITY OF WASHINGTON
* * * *
AMERICAN MATHEMATICAL SOCIETY \\ NAVAL WEAPONS CENTER
}

The Supporting Institutions listed above contribute to the cost of publication of this Journal, but they are not owners or publishers and have no responsibility for its content or policies.

Mathematical papers intended for publication in the Pacific Journal of Mathematics should be in typed form or offset-reproduced, (not dittoed), double spaced with large margins. Underline Greek letters in red, German in green, and script in blue. The first paragraph or two must be capable of being used separately as a synopsis of the entire paper. The editorial "we" must not be used in the synopsis, and items of the bibliography should not be cited there unless absolutely necessary, in which case they must be identified by author and Journal, rather than by item number. Manuscripts, in duplicate if possible, may be sent to any one of the four editors. Please classify according to the scheme of Math. Rev. Index to Vol. 39. All other communications to the editors should be addressed to the managing editor, Richard Arens, University of California, Los Angeles, California, 90024.

50 reprints are provided free for each article; additional copies may be obtained at cost in multiples of 50 .

The Pacific Journal of Mathematics is issued monthly as of January 1966. Regular subscription rate: $\$ 48.00$ a year (6 Vols., 12 issues). Special rate: $\$ 24.00$ a year to individual members of supporting institutions.

Subscriptions, orders for back numbers, and changes of address should be sent to Pacific Journal of Mathematics, 103 Highland Boulevard, Berkeley, California, 94708.

PUBLISHED BY PACIFIC JOURNAL OF MATHEMATICS, A NON-PROFIT CORPORATION

Printed at Kokusai Bunken Insatsusha (International Academic Printing Co., Ltd.), 270, 3-chome Totsuka-cho, Shinjuku-ku, Tokyo 160, Japan. 


\section{Pacific Journal of Mathematics}

\section{Vol. 46, No. 2 December, 1973}

Christopher Allday, Rational Whitehead products and a spectral sequence of

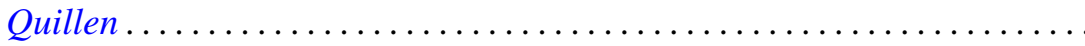

James Edward Arnold, Jr., Attaching Hurewicz fibrations with fiber

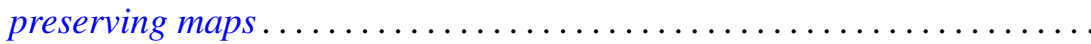

Catherine Bandle and Moshe Marcus, Radial averaging transformations with various metrics.................................

David Wilmot Barnette, A proof of the lower bound conjecture for convex

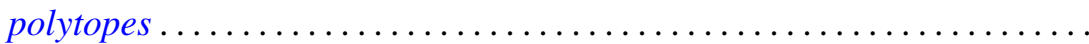

Louis Harvey Blake, Simple extensions of measures and the preservation of

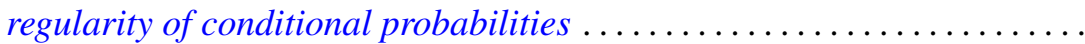

James W. Cannon, New proofs of Bing's approximation theorems for

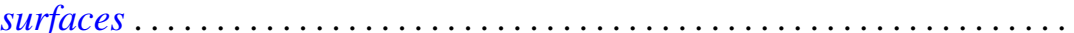

C. D. Feustel and Robert John Gregorac, On realizing HNN groups in

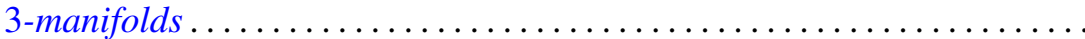

Theodore William Gamelin, Iversen's theorem and fiber algebras . . . . . . . . 389

Daniel H. Gottlieb, The total space of universal fibrations . . . . . . . . . . . .

Yoshimitsu Hasegawa, Integrability theorems for power series expansions of

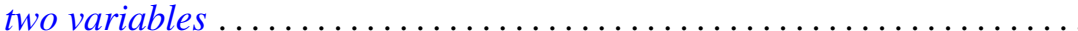

Dean Robert Hickerson, Length of period simple continued fraction expansion of $\sqrt{ } d$

Herbert Meyer Kamowitz, The spectra of endomorphisms of the disc algebra.

Dong S. Kim, Boundedly holomorphic convex domains

Daniel Ralph Lewis, Integral operators on $\mathscr{L}_{p}$-spaces ...

John Eldon Mack, Fields of topological spaces . . . . . . . . .

V. B. Moscatelli, On a problem of completion in bornology

Ellen Elizabeth Reed, Proximity convergence structures. .

Ronald C. Rosier, Dual spaces of certain vector sequence spaces .

Robert A. Rubin, Absolutely torsion-free rings

Leo Sario and Cecilia Wang, Radial quasiharmonic functions . .

James Henry Schmerl, Peano models with many generic classes .

H. J. Schmidt, The $\mathscr{F}$-depth of an $\mathscr{F}$-projector ............

Edward Silverman, Strong quasi-convexity. . . . . . . . . . . . . . . . . 549

Barry Simon, Uniform crossnorms ....................... 555

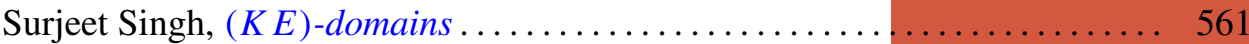

Ted Joe Suffridge, Starlike and convex maps in Banach spaces . . . . . . . . 575

Milton Don Ulmer, $C$-embedded $\Sigma$-spaces . . . . . . . . . . . . . . . . 591

Wolmer Vasconcelos, Conductor, projectivity and injectivity . . . . . . . . . 603 